\title{
Vaginal Hyperplasia and Progressive Vaginal Fold Prolapse in a Bullmastiff Bitch
}

\author{
Kenneth O. Anya1, Chike F. Oguejiofor ${ }^{1 *}$, Theophilus 0. Nnaji², Ikechukwu J. Udeani ${ }^{3}$ \\ ${ }^{1}$ Department of Veterinary Obstetrics and Reproductive Diseases, Faculty of Veterinary Medicine, University of Nigeria, \\ Nsukka, Nigeria \\ ${ }^{2}$ Department of Veterinary Surgery, Faculty of Veterinary Medicine, University of Nigeria, Nsukka, Nigeria \\ ${ }^{3}$ Veterinary Teaching Hospital, Faculty of Veterinary Medicine, University of Nigeria, Nsukka, Nigeria \\ Email: *chike.oguejiofor@unn.edu.ng
}

How to cite this paper: Anya, K.O., Oguejiofor, C.F., Nnaji, T.O. and Udeani, I.J. (2020) Vaginal Hyperplasia and Progressive Vaginal Fold Prolapse in a Bullmastiff Bitch. Open Journal of Veterinary Medicine, 10, 55-63.

https://doi.org/10.4236/ojvm.2020.105005

Received: February 24, 2020

Accepted: May 26, 2020

Published: May 29, 2020

Copyright () 2020 by author(s) and Scientific Research Publishing Inc. This work is licensed under the Creative Commons Attribution International License (CC BY 4.0).

http://creativecommons.org/licenses/by/4.0/ (c) (i) Open Access

\begin{abstract}
Canine reproductive problems constitute some of the most challenging cases encountered in small animal veterinary practice. This is usually complicated in breeding dogs by the unwillingness of clients to give consent for surgical interventions, due to the fear of loss of reproductive function. In this case, a two-year-old Bullmastiff bitch was presented to the Veterinary Teaching Hospital with a mass protrusion from the vulva. Clinical examination revealed an eversion of a tongue-shaped vaginal tissue from the floor of the vaginal wall which subsequently progressed to an eversion of the complete vaginal circumference forming a doughnut-shaped mass. Investigations carried out included ultrasonography, hematology, hormonal assay, vaginal cytology, vaginal swab microbial culture and antimicrobial sensitivity test. A diagnosis of vaginal fold prolapse (type III) which progressed from a type II prolapse was made. Due to the client's initial disinclination to a surgical intervention, the approach to the case evolved from a conservative management to an eventual surgical correction. There was a request to preserve the reproductive function of the bitch, therefore ovariohysterectomy was declined and the case was managed by surgical excision of the prolapsed vaginal mass under general anesthesia. A peri-vulvar purse string suture was placed temporarily to restrict any further prolapse. Histopathological evaluation of the excised vaginal tissue confirmed marked hyperplasia of the stratified squamous epithelium with intracellular edema and spongiosis. There was focal ulceration of vaginal mucosa with neutrophilic infiltration. The lamina propria showed reduced cellular density with loose and edematous connective tissue. Post-surgical care included daily care of surgical wound and the administration of analgesic, antibiotic and vitamin supplements. Subsequently, there was no recurrence of the condition in the bitch which came into estrus 27 weeks post-surgery, and was bred with successful conception.
\end{abstract}




\section{Keywords}

Vaginal Hyperplasia, Vaginal Fold Prolapse, Vaginal Surgery, Bitch, Estrus

\section{Introduction}

Reproductive tract anomalies in bitches are important causes of morbidity and infertility in dogs. Vaginal/vestibular masses can occur in both intact and spayed bitches of different breeds and may include vaginal prolapses, vaginal neoplasia, urethral neoplasia, and less commonly uterine prolapse, clitoral enlargement, vaginal polyps, vaginal abscessation, hematomas or retroflexion of the urinary bladder due to perineal hernia [1] [2].

Vaginal prolapse in the bitch has been referred to as vaginal hyperplasia, vaginal hypertrophy, estrual hypertrophy, vaginal eversion, vaginal protrusion or vaginal fold prolapse [1] [2] [3]. Vaginal fold prolapse (VFP) is the protrusion of edematous vaginal tissue through the opening of the vulva occurring as a result of an exaggerated response of the vaginal mucosa to estrogens [2] [4]. Clinically, the condition can be categorized into 3 different types based on the degree of vaginal tissue protrusion. Type I prolapse is characterized by a slight to moderate eversion of the vaginal floor to the external urethral orifice, which does not protrude through the vulva. In type II, there is complete prolapse through the vulva of a broad tongue-shaped or a dome-shaped mass originating from the floor of the vagina cranial to the urethral orifice. Type III is characterized by a complete prolapse of the entire circumference of the vaginal wall, which presents a doughnut-shaped or ring-shaped mass [1] [2] [3].

\section{Case Report}

A two-year-old Bullmastiff bitch was presented to the University Veterinary Teaching Hospital. The primary complaint was the protrusion of a mass from the vagina of the bitch.

Historical evaluation revealed that the condition started ten days ago when a bloody discharge from the vulva was observed. The client considered this to be a sign of estrus but by the following day, a protrusion from the vulva was observed. The condition then progressively increased in size until the time of hospital presentation. There was no previous record of pregnancy or occurrence of the condition. There was no record of any medical treatment or attempt to remedy the condition. A history of previous diet, illnesses, vaccinations and treatments were also noted.

The bitch had a body weight of $37 \mathrm{~kg}$, and showed anxiety with elevated physiologic parameters that included rectal temperature $\left(40.4^{\circ} \mathrm{C}\right.$; reference: $37.5^{\circ} \mathrm{C}$ $39.2^{\circ} \mathrm{C}$ ), pulse rate (156 beats/min; reference: $80-120$ beats $/ \mathrm{min}$ ) and respiratory rate (48 breaths/min; reference: 10 - 30 breaths $/ \mathrm{min}$ ). The lung sounds were normal following auscultation. The bitch was distressed and squatted frequently, 
suggesting signs of irritation. It was apparently in a good body condition with normal skin rebound. There was a slightly pink mucous membrane of the eyes. Palpable lymph nodes particularly the popliteal lymph nodes were slightly enlarged. There was presence of a few ectoparasites (ticks). Reproductive tract examination revealed an eversion of a tongue-shaped mass from the floor of the vagina cranial to the urethral orifice (Figure $1(\mathrm{~A})$ ). By day 7 post-presentation, the condition had progressed to an eversion of the complete vaginal circumference. There was eversion of the complete vaginal circumference forming an enlarged and turgid doughnut-shaped mass $14.5 \mathrm{~cm}$ in diameter with a lumen in the middle (Figure 1(B)). There was mild evidence of tissue trauma on the mucosa of the prolapsed vaginal tissue.

Clinical and laboratory investigations carried out included ultrasonography, hematology, hemoparasite evaluation, hormonal assay, vaginal cytology, vaginal histopathology, vaginal swab microbial culture and antimicrobial sensitivity test. Ultrasonography of the reproductive tract did not reveal any evidence of ovarian abnormalities, pregnancy or uterine prolapse. Hematological findings include a decrease in packed cell volume ( $\mathrm{PCV}=30 \%$; reference: $35 \%$ - 54\%), hemoglobin concentration $(\mathrm{Hb}=12.59 \mathrm{~g} / \mathrm{dl}$; reference: $13-19 \mathrm{~g} / \mathrm{dl})$ and red blood cell (RBC) count $\left(2.44 \times 10^{6} / \mu \mathrm{l}\right.$; reference: $\left.(4.9-7.9) \times 10^{6} / \mu \mathrm{l}\right)$. The total white blood cell (WBC) count was $11.80 \times 10^{3} / \mu \mathrm{l}$ (reference: $\left.(6-17) \times 10^{3} / \mu \mathrm{l}\right)$ while the differential WBC counts were lymphocyte (48\%; reference: $12 \%-30 \%)$, neutrophil (52\%; reference: $58 \%-85 \%)$, monocyte ( $0 \%$; reference: $2 \%$ - 10\%), eosinophil (0\%; reference: $0 \%-9 \%)$, and basophil ( $0 \%$; reference: $0 \%-1 \%)$. No hemoparasites (Trypanosoma sp., Babesia sp.) were observed in the blood. Serum concentrations of steroid hormones were measured using enzyme immunoassay (EIA) test kits (Monobind; Lake Forest, CA, USA) and a microplate reader (StatFax 4200; Awareness Tech, Palm City, FL, USA). Hormonal evaluation revealed a serum progesterone concentration of $3.71 \mathrm{ng} / \mathrm{ml}$ and oestradiol concentration of $22.09 \mathrm{pg} / \mathrm{ml}$. Daily vaginal cytology was performed to monitor the progressive
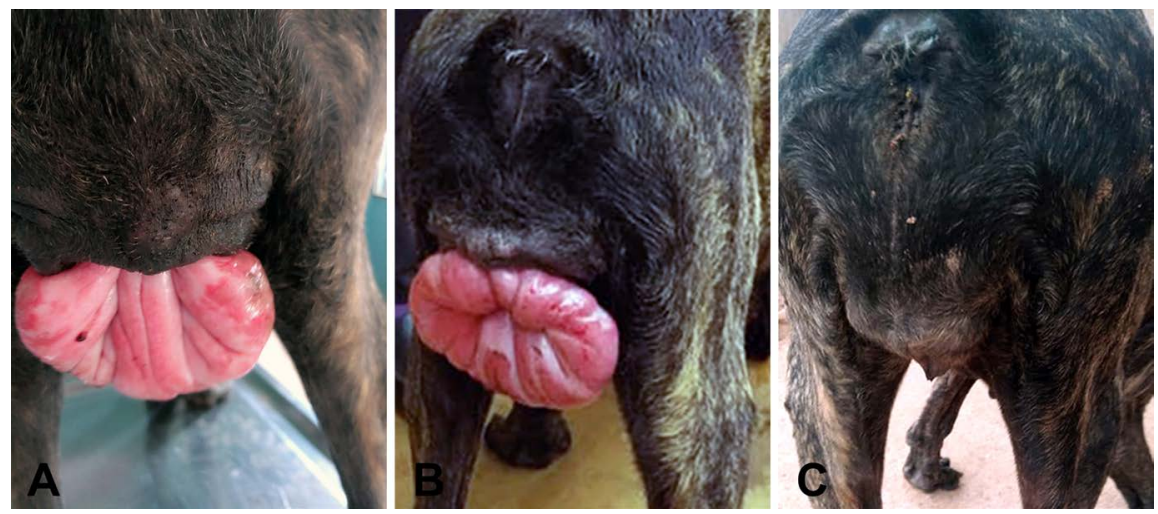

Figure 1. Surgical correction of a progressive vaginal fold prolapse in a Bullmastiff bitch. A: note the eversion of a broad tongue-shaped mass from the floor of the vagina (type II vaginal fold prolapse) which progressed to $B$ : eversion of complete vaginal circumference forming a doughnut-shaped mass (type III vaginal fold prolapse). C: patient recovery 14 days post-surgery. 
hormonal effect on the reproductive tract. There was a steady state of $100 \%$ enucleate superficial (cornified) vaginal epithelial cells indicating an estrus phase of the reproductive cycle (Figure 2). By day 7 post-presentation, there was a change of the cytology from $100 \%$ cornification (estrus) to a mixture of superficial and intermediate/parabasal cells and reappearance of WBCs in the smear (signifying the first day of diestrus). Microbial culture of the vaginal swab for 24 $\mathrm{h}$ yielded a scanty growth of Staphylococcus aureus that was sensitive to all the antibiotics tested. Based on the history and clinical and laboratory findings, a diagnosis of vaginal fold prolapse (type III) which progressed from a type II prolapse was made.

The patient was initially stabilized and treated for mild anemia by placement on daily balanced ration, and by administering Iron dextran (Hexin ${ }^{\otimes}$, HebeiKexing Pharmaceutical, Hebei, China) $2 \mathrm{ml}$ intra-muscularly (IM) and repeated after 3 days, and Multivitamin (Multivit ${ }^{\circledR}$, Bioveta, Komenského, Czech Republic) $2 \mathrm{ml} \mathrm{IM}$ once daily for 5 days. Tick infestation was controlled by dusting with pyrethrine powder (BioQarah, Lagos, Nigeria). The client disinclined surgical treatment so the initial approach was a conservative management of the case. The patient was fixed with an Elizabethan collar; the prolapsed tissue was cleaned and managed daily while waiting to see if a spontaneous regression would occur following an expected decrease in estrogen level as the bitch progressed from estrus to diestrus. However, the condition failed to regress by day 7 post-presentation but progressed from a type II to a type III prolapse. Therefore, a surgical approach to the case was recommended. Following due consultation with the client, there was need to preserve the reproductive function of the bitch. Thus ovariohysterectomy was declined and the case was managed by surgical excision of the prolapsed vaginal mass under general anesthesia. Prior to surgery, hematological evaluation revealed improved PCV (37\%), Hb (13.28 g/dl), $\mathrm{RBC}$ count $\left(5.31 \times 10^{6} / \mu \mathrm{l}\right)$ and normal WBC count $\left(11.0 \times 10^{3} / \mu \mathrm{l}\right)$.

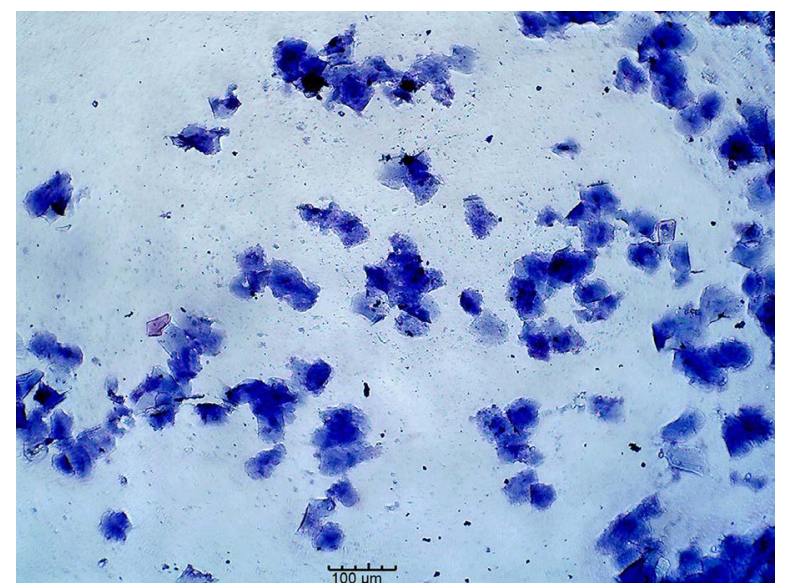

Figure 2. Vaginal cytology micrograph from a Bullmastiff bitch with vaginal fold prolapse. There was a predominance of enucleate superficial (cornified) epithelial cells indicating an estrus phase of the cycle. (Leishman's staining; magnification $=100 \times$; scale bar $=100 \mu \mathrm{m})$. 
The vulvar area was shaved and scrubbed with $0.3 \%$ chlorhexidine gluconate (Purit ${ }^{\oplus}$, SaroLifecare Ltd., Ibadan, Nigeria). Premedication was performed using atropine sulfate (Pauco Atropine $e^{\oplus}$ Jiangsu Huayang Pharmaceutical, Jiangsu, China) at $0.04 \mathrm{mg} / \mathrm{kg}$ b.w., IM and xylazine hydrochloride (AnaSed ${ }^{\oplus}$, Akorn, Lake Forest, IL, USA) at $0.5 \mathrm{mg} / \mathrm{kg}$ b.w., IM and general anesthesia using ketamine hydrochloride (Ketmin ${ }^{\oplus}$, Laborate, Panipat, India) at $5 \mathrm{mg} / \mathrm{kg}$ b.w., IM. The patient was placed on dorsal recumbency and intubated. An infusion line of Ringer's lactate solution (Dana ${ }^{\oplus}$, Dana Pharmaceutical, Ibadan, Nigeria) was set intra-operatively and delivered at $0.15 \mathrm{ml} / \mathrm{kg}$ b.w. $/ \mathrm{min}$. The urethra was catheterized using a size 8 urinary catheter to avoid damage to the urethra during surgery. Artery forceps were used to clamp the everted vaginal mass. Hemorrhage was controlled as previously described [5] by placing ten circumferential ligatures from the outside passing through lumen and out through the wall of the everted mass using chromic catgut size 2 - 0 to ligate the major blood vessels of the vaginal wall. The everted vaginal tissue was cut off while taking care to check for hemorrhage. The cut edges of the vaginal wall were apposed using chromic catgut size $2-0$ in a simple interrupted suture pattern. The vaginal stump was cleaned of blood clots and checked carefully for hemorrhage before being replaced in the vagina, and the urinary catheter removed. A purse string suture was placed around the vulvar opening using nylon suture size $2-0$ to narrow the vulvar canal and restrict any further prolapse. The vulvar canal was checked for patency to allow for normal passage of urine from the tract. The patient recovered uneventfully from the general anesthesia.

A sample of the excised vaginal tissue was fixed in $10 \%$ phosphate-buffered formalin and sent for histopathology. Vaginal histopathology confirmed hyperplasia of the stratified squamous epithelium with intracellular edema and spongiosis. There was focal ulceration of vaginal mucosa with neutrophilic infiltration. The lamina propria showed reduced cellular density with loose and edematous connective tissue (Figure 3).

Post-surgical care included daily cleaning and care of surgical wound, daily treatment with antibiotics, analgesic, and multivitamins. The patient was administered with procaine penicillin G at 10,000 IU/kg b.w.; Benzathine penicillin G at 10,000 IU/kg b.w. and Dihydrostreptomycinsulphate at $20 \mathrm{mg} / \mathrm{kg}$ b.w. (Procilline LA ${ }^{\oplus}$, Kepro, Deventer, The Netherlands) IM once and repeated after 3 days; Tramadol hydrochloride (Tradyl ${ }^{\oplus}$, PT Interbat, East Java, Indonesia) at 2.5 mg/kg b.w., IM twice daily and Multivitamin (Multivit ${ }^{\oplus}$, Bioveta, Komenského, Czech Republic) $2 \mathrm{ml}$ IM once daily for 5 days, followed by one Multivitamin tablet (K-9 Favorite ${ }^{\circledast}$, Shijiazhuang Bochuang, Shijiazhuang, China) orally daily for 7 days. An Elizabethan collar was placed to prevent self-mutilation by the patient. The patient's urination was observed daily to ensure no surgical complication or damage to the urethra or the urethral orifice. The patient made a good recovery. Wound healing progressed adequately and the purse-string sutures were removed 7 days post-surgery. The patient was discharged uneventfully 14 days post-surgery (Figure $1(C)$ ). Subsequently, there was no recurrence of the 


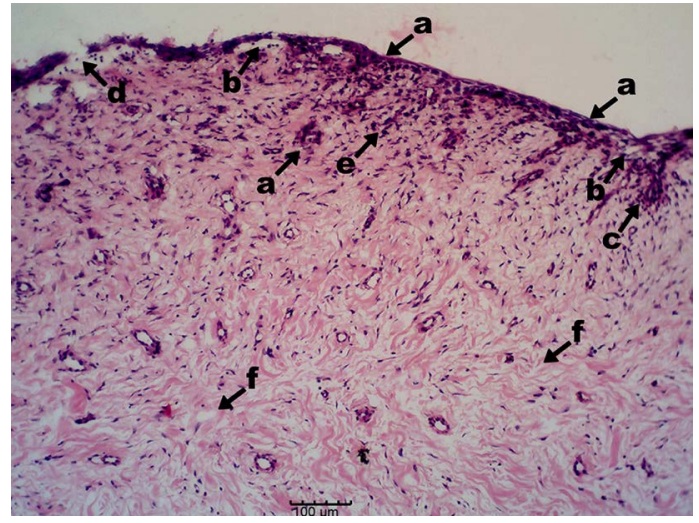

Figure 3. Histopathological micrograph of the everted vaginal tissue excised from a Bullmastiff bitch with vaginal fold prolapse. a-Hyperplasia of the stratified squamous epithelium with cells showing hyperchromasia and little cytoplasm. b-Intracellular edema and spongiosis in the vaginal epithelium with the presence of intra-epidermal vesicles. c-Downward hyperplastic growth of vaginal epithelium into underlying submucosa. d-Focal area of ulceration of vaginal mucosa with neutrophilic infiltration. e-Vaginal submucosa composed of numerous spindle-shaped cells with numerous blood vessels. f-Lamina propria showing reduced cellular density with loose and edematous connective tissue. $(\mathrm{H}$ and $\mathrm{E}$ staining; magnification $=\times 100$; scale bar $=100 \mu \mathrm{m}$ ).

condition in the bitch which came into estrus 27 weeks post-surgery, and was bred with successful conception.

\section{Discussion}

Canine reproductive problems constitute some of the most challenging cases encountered in small animal veterinary practice. This is usually complicated in breeding dogs by the unwillingness of clients to give consent for surgical interventions due to the fear of loss of reproductive function. This concern is also amplified in exotic large dog breeds that usually have long (6 - 12 months) inter-oestrus interval. The client is thus anxious to breed the bitch for maximal economic returns before the decline in reproductive efficiency. This report described the evaluation, diagnosis and treatment of a case of vaginal hyperplasia and progressive vaginal fold prolapse in a two-year-old Bullmastiff bitch. Due to the client's initial disinclination to a surgical intervention, the approach to the case evolved from a conservative management to an eventual surgical correction.

Estrogen secretion from hormonally-active ovarian cysts or ovarian tumors (e.g. granulosa cell tumors) may induce persistent estrus in affected bitches [6]. However, these were not considered as causative factors following ultrasonography and sequential vaginal cytology. Instead there were vaginal cytological changes in response to hormonal transition from the estrus to the diestrus phase of the cycle. The failure of the everted mass to regress following diestrus ruled out estrual hypertrophy. Histopathological findings demonstrated vaginal hyperplasia but there was no evidence of dysplasia or neoplastic tissue.

A previous review reported a histopathologic demonstration of marked oedema and fibroplasia of the vaginal mucosa rather than hyperplasia or hyper- 
trophy [2]. Our findings differed from this, in line with a recent report of significant differences in histomorphologic alterations in excised vaginal tissues from different breeds of bitches [7]. We did not observe marked vaginal cornification and stratification; instead there was degenerative changes of the vaginal mucosa with spongiosis and ulceration of the mucosa, downward hyperplastic growth of the epithelium and loose and edematous connective tissue of the submucosa.

The etiology of the prolapse in this case may be related to the patient's age, breed and response to estrogenic stimulation during estrus. VFP is most commonly observed in young bitches (usually between 1.5 - 2.7 years) during the follicular stage of the first to third oestrous cycle [2]. VFP has also been commonly reported in brachycephalic breeds or crosses including the Bullmastiff [8] [9]. Although not clearly established, brachycephalic breeds are thought to be genetically predisposed to VFP, and may have a weakness of the peri-vulvar tissue [10]. The presence of cornified vaginal epithelium together with the observed serum levels of oestradiol and progesterone indicated that the bitch was in estrus [11]. The oestradiol level was also within the range reported for bitches in the estrus phase [11] thus suggesting that condition may also have resulted from an exaggerated response of the patient to normal estrogenic activity during this period. Elevated serum estrogen concentrations during the follicular phase of the oestrous cycle causes edema of the submucosa and stratified squamous epithelium lining of the vagina. This has been associated with a high incidence of the disorder during pro-estrus/estrus (up to 86\%) and late pregnancy [1] [2].

It was important that the client presented the bitch for treatment. If left uncorrected, the prolapsed tissue is vulnerable to self-mutilation and tissue damage that can lead to hemorrhage, infection and necrosis. These possible complications can influence the treatment choice and prognosis of the condition. Options for the treatment of vaginal prolapse in bitches may include a conservative approach (observation or hormonal treatment of the bitch until regression of the condition post-estrus), surgical excision of the prolapsed tissue and/or ovariohysterectomy. Uncomplicated type I and II vaginal prolapses in bitches in estrus may resolve spontaneously following the fall in estrogen concentrations in diestrus [2]. However, there was progression to an extensive tissue eversion (type III prolapse) in this case, and a failure of tissue regression post-estrus. The client was also informed of the possibility of future recurrence of the condition following a conservative management. There are reports of a high incidence $(66 \%-100 \%)$ of recurrence of vaginal prolapse in affected bitches that received conservative treatment [2] [7]. Ovariohysterectomy offers a permanent treatment method and prevention of vaginal prolapse [1]. However, the client emphasized that the bitch was kept primarily for breeding, and demanded the preservation of the reproductive function of the patient. Ovariohysterectomy was therefore not considered and surgical excision of the prolapsed vaginal mass was held as the treatment option in the case.

The surgery was performed under general anesthesia due to extensive nature of the tissue prolapse (Type III) which involved significant tissue cutting and 
suturing. The everted vaginal tissue was also highly vascularized necessitating careful placement of ligatures to prevent undue hemorrhage. A purse string suture placed around the vulvar opening was used to retain the vaginal tissue stump to prevent further tissue protrusion, and to allow for proper healing of the surgical wound. A systemic antibiotic cover was also given to minimize the risk of post-surgical infection and complications. Analgesia was important to relieve pain associated with surgery, and to improve animal welfare. Vitamin supplementation would help to boost the patient's appetite, energy and nutrient metabolism, immune response and wound healing. The use of an Elizabethan collar post-surgery was useful in preventing self-mutilation by the patient and postsurgical infection and complications.

\section{Conclusion}

In summary, reproductive cases in breeding bitches are often complicated by clients' refusal of surgical intervention. It is important to respect the client's opinion but also to discuss the possible limitations of conservative management. A surgical excision of the prolapsed vagina was successful in this case and ovariohysterectomy was avoided thereby preserving the reproductive ability of the bitch. Moreover, histopathological findings from the excised vaginal tissue provided evidence of vaginal mucosal spongiosis, ulceration and hyperplasia rather than marked vaginal cornification and stratification.

\section{Acknowledgements}

The authors are grateful to Justina I. Oguejiofor of Projex Biomedical Laboratory, Nsukka for the serum hormonal analysis.

\section{Conflicts of Interest}

The authors declare no conflicts of interest regarding the publication of this paper.

\section{References}

[1] Manothaiudom, K. and Johnston, S.D. (1991) Clinical Approach to Vaginal and Vestibular Masses in the Bitch. Veterinary Clinics of North America: Small Animal Practice, 21, 509-521. https://doi.org/10.1016/S0195-5616(91)50057-7

[2] Sontas, H.B., Ekici, H. and Romagnoli, S. (2010) Canine Vaginal Fold Prolapse: A Comprehensive Literature Review. European Journal of Companion Animal Practice, 20, 127-135.

[3] Schutte, A.P. (1967) Vaginal Prolapse in the Bitch. Journal of South African Veterinary Association, 38, 197-203.

[4] Alan, M., Cetin, Y., Sendag, S. and Eski, F. (2007) True Vaginal Prolapse in a Bitch. Animal Reproduction Science, 100, 411-414. https://doi.org/10.1016/j.anireprosci.2006.10.022

[5] Antonov, A.L., Atanassov, A.S. and Georgiev, P.I. (2009) A Modified Technique for Prolapsed Fold Excision in a Bitch with Vaginal Hyperplasia. Bulgarian Journal of 
Veterinary Medicine, 12, 260-264.

[6] Johnston, S.D., Kustritz, M.V.R. and Olson, P.N.S. (2001) Disorders of Canine Ovary. In: Canine and Feline Theriogenology, WB Saunders, Philadelphia, 193-205.

[7] Galal, S.M., Fathi, M., Ismail, S.T., El Belely, M.S. and Mohamed, F.F. (2018) Clinical Diagnosis and Surgical Approaches of Vaginal Hyperplasia in Bitches. Asian Pacific Journal of Reproduction, 7, 220-224.

[8] Johnston, S.D., Kustritz, M.V.R. and Olson, P.N.S. (2001) Vaginal Prolapse. In: Canine and Feline Theriogenology, WB Saunders, Philadelphia, 233-235.

[9] Feldman, E.C. and Nelson, R.W. (2004) Vaginal Defects, Vaginitis and Vaginal Infection. In: Canine and Feline Endocrinology and Reproduction, 3rd Edition, WB Saunders, Philadelphia, 901-918.

[10] Wykes, P.M. (1986) Diseases of the Vagina and Vulva in the Bitch. In: Current Therapy in Theriogenology, WB Saunders, London, 476-481.

[11] Blendinger, K. (2007) Physiology and Pathology of the Estrous Cycle of the Bitch. Proceedings of the 56th Congresso Internazinale Multisala (SCIVAC), Rimini, 73-77. 